\title{
PREVALENCIA DE COLONIZACIÓN POR STREPTOCOCCUS AGALACTIAE (GRUPO B) DURANTE EL EMBARAZO PESQUISADO EN MEDIO DE CULTIVO SELEC TIVO
}

\author{
Enrique Valdés $R .^{1}$, Carolina Pastene $S{ }^{1}$, Alejandro Morales Pa, Bárbara Gutiérrez R. ${ }^{a}$, \\ Ana Canales P. ${ }^{b}$, Pabla Martínez O. ${ }^{b}$, Guido Juarez D. ${ }^{1}$, Rafael Caballero T. ${ }^{1}$ \\ ${ }^{1}$ Departamento de Obstetricia y Ginecología. Hospital Clínico U. de Chile
}

anternos de Medicina, Universidad de Chile

bTecnólogos Médicos, Laboratorio Central, Unidad de Microbiología, Hospital Clínico Universidad de Chile

\section{RESUMEN}

Streptococcus agalactiae es el principal agente bacteriano responsable de la sepsis neonatal. Para evitar la infección perinatal se recomienda su pesquisa en la región vagino-anal durante el tercer trimestre, y tratamiento antibiótico durante el trabajo de parto en las gestantes colonizadas. El objetivo de este estudio es conocer la prevalencia de colonización del Streptococcus agalactie en la población de embarazadas controladas en la maternidad del Hospital Clínico de la Universidad de Chile, en el período comprendido entre el 1 de marzo y el 31 de mayo de 2003. Se tomó cultivo selectivo de Todd Hewitt, entre las 35 y 37 semanas de gestación a 185 embarazadas. Se determinó una prevalencia de colonización vagino-anal de 14,0\%.

\section{PALABRAS CLAVE: Streptococcus agalactiae, sepsis neonatal}

\section{SUMMARY}

Streptococcus agalactiae is the main bacterial agent in neonatal sepsis. To avoid the perinatal infection, a vaginal and anal culture in the third trimester is recommended and then treated with antibiotics during labour. The aim of the study was to study the prevalence of Streptococcus agalactiae in pregnant patients in University of Chile Hospital in Santiago. The study period was from March 1 to May 31 of 2003. Vaginal and anal samples were taken at 35-37 weeks using selective medium (Todd Hewitt broth). A total of 185 patients were studied and the prevalence of streptococcus was $14,0 \%$.

\section{KEY WORDS: Streptococcus agalactiae, neonatal sepsis}

\section{INTRODUCCIÓN}

El streptoccoccus agalactiae $\beta$ hemolítico Grupo B (SGB), es el principal agente etiológico de sepsis neonatal precoz la que tiene una frecuencia entre 1 a $4 / 1000$ nacidos vivos y con una letalidad estimada entre 5 a 20\% (1,2). Además, es uno de los microorganismos más frecuentemente involucrados en complicaciones infecciosas del embarazo, parto y puerperio, como infección urinaria, corioamnionitis y endometritis puerperal (3-5). 
Como respuesta a esta situación el Colegio Americano de Obstetricia y Ginecología (ACOG), la Academia Americana de Pediatría (AAP) y el Centro de Control de Enfermedades de Estados Unidos (CDC) han aconsejado el uso de profilaxis antibiótica durante el trabajo de parto, a través de dos protocolos con el objetivo de disminuir la incidencia de infección neonatal precoz.

1) Embarazadas en trabajo de parto, con factores de riesgo de infección: prematurez, síndrome febril en el trabajo de parto, antecedentes de sepsis neonatal por SGB, bacteriuria asintomática por SGB y la rotura prematura de las membranas por más de 12 horas, sin toma de cultivos previos (1).

2) Embarazadas con factores de riesgo y las portadoras de SGB pesquisadas por cultivo en el tercer trimestre del embarazo (35-37 semanas); avalado por que la sepsis perinatal se presenta entre un 30 a $70 \%$ en recién nacidos sin "factores de riesgo" $(6,7)$.

Este último protocolo ha logrado obtener las menores tasas de sepsis neonatal precoz $(0,1$ a $1 / 1000$ nacidos vivos) (8). Sin embargo, el rendimiento de la pesquisa depende tanto de las zonas cultivadas como de la calidad de los medios de cultivos. Una experiencia reciente realizada por los autores con muestras vaginales y perianales, sembradas en medio de cultivo corriente, identificó una prevalencia de $6,2 \%$ de embarazadas portadoras (9), cifra inferior a otras series nacionales, que con medios selectivos obtuvieron una prevalencia de $20 \%$ de portadoras (10-12). De ahí que se considere el cultivo vagino-perianal sembrado en medio selectivo de Todd-Hewitt como el estándar para el diagnóstico de portación de SGB en el embarazo, que permite identificar prevalencias de hasta $40 \%(3,13)$.

Los objetivos de este estudio fueron: determinar la prevalencia de SGB utilizando un medio selectivo, evaluar el impacto de la profilaxis antibiótica en la prevención de sepsis neonatal precoz y establecer la sensibilidad antibiótica del SGB, en una población de embarazadas atendidas en la maternidad del Hospital Clínico de la Universidad de Chile.

\section{MATERIAL Y MÉTODO}

Se diseñó un estudio retrospectivo para determinar la prevalencia de portadoras de Streptococo agalactiae (SGB). Para ese objetivo se procede a extraer desde la base de datos del Servicio de Microbiología del Hospital Clínico de la Universidad de Chile, los resultados de los cultivos vagino- perianales, tomados de embarazadas que cursan un embarazo entre las 35 a 37 semanas, según norma del Servicio de Obstetricia de dicho centro. Las muestras fueron sembradas en cultivo selectivo de Todd Hewitt, suplementado con ácido nalidíxico y gentamicina, e incubados a $37^{\circ}$ por 24 horas. El estudio se efectuó en el período comprendido entre el $1^{\circ}$ de marzo y el 31 de mayo de 2003. En las muestras positivas se procedió al estudio de sensibilidad antibiótica.

Las embarazadas con cultivos positivos, como las con factores de riesgo, recibieron al ingreso en trabajo de parto, 2 gramos de ampicilina EV como dosis de carga, seguido de 1 gramo cada 4 horas hasta la resolución del parto; las con antecedentes de alergia a penicilina recibieron $600 \mathrm{mg}$ de clindamicina EV seguido por $300 \mathrm{mg}$ cada 4 horas.

Se efectuó seguimiento de los neonatos de madres portadoras y de los recién nacidos con diagnóstico de infección neonatal, revisando la base de datos del Servicio de Neonatología para determinar la prevalencia de sepsis neonatal precoz por SGB antes de los 10 días de vida y la efectividad de la profilaxis antibiótica en los meses señalados.

\section{RESULTADOS}

Se controlaron 206 embarazadas entre las 35 y 37 semanas en el período estudiado, efectuándose cultivos vagino-perianales a 185 pacientes $(89,8 \%$ de cobertura). En 26 de ellas se identificó SGB lo que determina una prevalencia de $14,0 \%$.

Del total de pacientes con cultivos positivos, 11 eran portadoras de SGB en la muestra vaginal $(42,3 \%)$, 8 en la muestra perianal $(30,8 \%$ ) y 7 en ambas muestras (26,9\%) (Tabla I).

El antibiograma de las cepas cultivadas mostró $100 \%$ de sensibilidad para penicilina, ampicilina y eritromicina y $70,5 \%$ para lincomicina.

Todas las embarazadas portadoras de SGB y las con factores de riesgo de transmisión vertical

Tabla I

CULTIVOS POSITIVOS DE STREPTOCOCO GRUPO B EN 185 EMBARAZADAS EN EL TERCER TRIMESTRE

\begin{tabular}{lcc}
\hline Sitio de cultivo & $n$ & $\%$ \\
\hline Vaginal & 11 & 42,3 \\
Anal & 8 & 30,8 \\
Vaginal + anal & 7 & 26,9 \\
\hline Total & 26 & 100 \\
\hline
\end{tabular}


recibieron profilaxis antibiótica durante el trabajo de parto.

De los 206 nacidos vivos que ingresaron al estudio, 6 presentaron diagnóstico de sospecha de sepsis neonatal precoz. Uno tuvo hemocultivos positivos para SGB $(0,5 \%)$, destacamos que su madre era portadora de la bacteria y recibió profilaxis antibiótica durante el trabajo de parto.

\section{DISCUSIÓN}

Los estudios de prevalencia de SGB en embarazadas muestran tasas de colonización que varía entre 5 y $25 \%(9,11)$, dependiendo de la población estudiada, la ubicación geográfica, la región anatómica de obtención de la muestra (vaginal y/o anal) y el medio de cultivo utilizado (selectivo y no selectivo).

Este estudio permite enfatizar la necesidad del uso de medios de cultivos selectivos en la pesquisa del SGB, dado que en poblaciones gestantes de similares características demográficas, la prevalencia utilizando un medio no selectivo fue de $6,2 \%(9)$; al introducir un medio selectivo la prevalencia subió a $14 \%$, experiencia similar a la señalada por el grupo de Abarzúa y cols que al sembrar en un medio corriente con muestras exclusivamente vaginales obtuvo una prevalencia de $5 \%$ y al introducir el cultivo selectivo se elevó a 19,8\% (12).

El caldo de cultivo Todd Hewitt como medio selectivo cumple con las exigencias necesarias para el desarrollo de SGB, ya que cuenta en su composición con ácido nalidíxico y gentamicina, antibióticos que inhiben el desarrollo de otros gérmenes y que pueden interferir en el aislamiento.

Este estudio ratifica la importancia de la toma de cultivos de la región vaginal y anal, ya que en un porcentaje importante de éstos el SGB se aisló sólo en la muestra anal $(30,8 \%)$, esto significa que la toma vaginal única deja casi un tercio de las embarazadas en riesgo de transmisión vertical al no recibir profilaxis.

El esfuerzo para pesquisar al SGB tiene como fin disminuir la incidencia de sepsis neonatal pre$\mathrm{coz}$, para lo cual cada centro debe contar con una adecuada profilaxis antibiótica para sus embarazadas durante el trabajo de parto. Esto hace necesario conocer la sensibilidad antibiótica del germen en esa población. En este estudio todas las cepas aisladas presentaron sensibilidad a ampicilina y penicilina, ambos antibióticos han sido sugeridos como tratamiento de elección (14), y concuerda con lo informado por otro centro nacional (11).
Respecto a la sepsis neonatal y su gran impacto en salud pública debido a la elevada tasa de mortalidad neonatal (58 a 71\%), lo único que ha demostrado efectividad es la administración de antibióticos desde el inicio del trabajo de parto. La estrategia más efectiva es la que considera a las embarazadas con factores de riesgo y la población de bajo riesgo con cultivos positivos y que son tratadas profilácticamente durante el trabajo de parto $(1,6,7,14,15)$.

Llama la atención el caso de sepsis neonatal presentado pese a que la madre recibió tratamiento profiláctico completo con ampicilina, evento que podría ser explicado por una carga bacteriana mayor, o por paso del germen a través de las membranas con desarrollo de una forma subclínica de corioamnionitis, o la falta de tiempo necesario para alcanzar el antibiótico una concentración inhibitoria mínima adecuada. A pesar de lo anterior la prevalencia de sepsis neonatal precoz fue similar a lo publicado en series donde se empleó este protocolo $(4,8 / 1000 \mathrm{RN})$.

Se concluye que la reducción de la morbimortalidad por sepsis neonatal precoz es posible mediante la identificación del SGB entre las 35 y 37 semanas de amenorrea, mediante cultivos selectivos tomados desde la vagina y el ano, con el posterior tratamiento antibiótico profiláctico de las embarazadas portadoras al ingreso en trabajo de parto.

\section{BIBLIOGRAFÍA}

1. CDC. Adoption of hospital polices for prevention of perinatal group B streptococcal disease. United States, MMWR 1998; 47(32): 665-70.

2. Tapia JL, Prado P. Ventura-Juncá P. Infecciones bacterianas. En: Manual de Neonatología, Tapia JL, Ventura-Juncá P (eds). Editorial Mediterráneo, 2000; Cap 25; 227-40.

3. Winn H, Hobbins J. Clinical Maternal-Fetal Medicine. The Parthenon Publishing Group, 2000; Cap 19: 209-11.

4. Faro S. Group B beta-hemolytic streptococci and puerperal infections. Am J Obstet Gynecol 1980; 139: 686-89.

5. Stoll BJ, Schuchat A. Maternal carriage of group B streptococci in developing countries. Pediatr Infect Dis J 1988; 17: 499-503.

6. Schrag S, Gorwitz R, Fultz-Butts P, Schuchat A. Prevention of perinatal group streptococcal disease. Revised guidelines from CDC. MMWR Recomm Rep 2002; 51 (RR-11): 1-22.

7. Rouse DJ, Cliver SP, Cutter GR. Strategies for the prevention of early-onset neonatal group B streptococcal sepsis: a decision analysis. Obstet Gynecol 1994; 83(4): 483-94. 
8. Yancey M, Duff P: An analysis of the cost-efectiveness of the select protocols for the prevention of the neonatal group B streptococcal infection. Obstet Gynecol 1994; 83(3): 367-71.

9. Valdés E, Pastene C, Grau M, Catalán J, Candia P, Juarez G, Caballero R. Prevalencia de colonización por Streptococcus agalactiae (grupo B) en el tercer trimestre del embarazo en medio de cultivo no selectivo. Rev Chil Obstet Ginecol 2003; 68(4): 30508.

10. Guzmán AM, Abarzúa F, Belmar C, García P, Rioseco A, Oyarzún E: Resultados de la aplicación del protocolo basado en screening para la búsqueda de streptococcus agalactiae en el tercer trimestre del embarazo. Rev Chil Infectol 2001; 18(3): 187-92.

11. Belmar C, Abarzúa F, Becker J, Guzmán AM, García P, Oyarzún E. Estudio de sensibilidad antimicrobiana de 183 cepas de Streptococcus agalactiae aisladas en región vagino perineal de embarazadas en el tercer trimestre. ReV ChIL OBSTET GINECOL 2002; 67 (2): 106-09.

12. Abarzúa F, Guzmán A, Belmar C, Becker J, García P, Rioseco A, Oyarzún E. Prevalencia de colonización por Streptococcus agalactiae (grupo B) en el tercer trimestre del embarazo, evaluación del cultivo selectivo, experiencia en 2192 pacientes. REV CHIL OBSTET GINECOL 2002; 67(2): 89-93.

13. Anthony BS, Okaba DM, Hobel CJ, Epidemiology of Group B streptococcus: Longuitudinal observation during pregnancy. J Infect Dis 1978; 137: 524-30.

14. CDC: Decreasing incidence of perinatal group $B$ streptococcal disease- United States, 1993-1995. MMWR 1997; 46(21): 473-77.

15. Main EK, Slagle T. Prevention of early-onset invasive neonatal group streptococcal disease in private hospital setting: the superiority of culture-based protocols. Am J Obstet Gynecol 2000; 182(6): 1344-54. 